\title{
On the Hardness of Range Assignment Problems
}

\author{
Bernhard Fuchs* \\ Center for Applied Computer Science Cologne (ZAIK) \\ University of Cologne, Weyertal 80, 50931 Köln, Germany.
}

\begin{abstract}
We investigate the computational hardness of the ConneCtivity, the Strong Connectivity and the Broadcast type of Range Assignment Problems in $\mathbb{R}^{2}$ and $\mathbb{R}^{3}$. We present new reductions for the CONNECTIVITY problem, which are easily adapted to suit the other two problems. All reductions are considerably simpler than the technically quite involved ones used in earlier works on these problems. Using our constructions, we can for the first time prove NP-hardness of these problems for all real distance-power gradients $\alpha>0$ (resp. $\alpha>1$ for BROADCAST) in 2-d, and give improved lower bounds on the approximation ratios of all three problems in 3-d for all $\alpha>1$. In particular, we derive the overall first APX-hardness proof for BROADCAST. This was an open problem posed in earlier work in this area, as was the question whether (Strong) Connectivity remains NP-hard for $\alpha=1$. Additionally, we give the first hardness results for so-called well-spread instances.
\end{abstract}

\section{Introduction}

\subsection{Problem definition}

Let $S$ be a finite set of $n$ points in the Euclidean space $\mathbb{R}^{d}$. A range assignment for $S$ is a function $r: S \rightarrow \mathbb{R}_{+}$. For convenience, we write $r_{v}$ instead of $r(v)$. The cost of a range assignment is defined as

$$
\operatorname{cost}(r)=\sum_{v \in S} r_{v}^{\alpha}
$$

for some real constant $\alpha>0$.

The underlying intuition is that the elements of $S$ are given radio stations, and one can choose for each station $v \in S$ a corresponding data transmission range (radius) $r_{v}$. Sending data at radius $r_{v}$ consumes energy proportional to $r_{v}^{\alpha}$. A prominent example for this setting is a wireless network. The parameter $\alpha$ is called the distance-power gradient,

\footnotetext{
*Email: bfuchs@zpr.uni-koeln.de
} 
and realistic values for $\alpha$ range from 1 to more than 6 , according to [13], where the reader is directed to for further reading on the background behind this model.

Let $\operatorname{dist}(u, v)$ denote the Euclidean distance between two points $u, v \in \mathbb{R}^{n}$. A range assignment $r$ directly defines two kinds of graphs, which reflect communication properties of $r$ :

- Let $\vec{G}_{r}=\left(S, A_{r}\right)$ be the directed communication graph of range assignment $r$, where an $\operatorname{arc}(u, v)$ is contained in $A_{r}$ iff $u$ can send to $v$, i.e., the radius of $u$ is at least as large as the distance between $u$ and $v$. In short, $(u, v) \in A_{r} \Leftrightarrow r_{u} \geq \operatorname{dist}(u, v)$.

- Let $G_{r}=\left(S, E_{r}\right)$ be the undirected communication graph of range assignment $r$. Here, an edge $\{u, v\}$ is included in $E_{r}$ iff $u$ can send to $v$ and $v$ can send to $u$, i.e., $\{u, v\} \in E_{r} \Leftrightarrow \min \left\{r_{u}, r_{v}\right\} \geq \operatorname{dist}(u, v)$. In other words, $G_{r}$ contains exactly the antiparallel arcs of $\vec{G}_{r}$.

We can now define which kinds of requirements we might demand of a range assignment $r$ :

- Connectivity (C): $G_{r}$ must be connected.

- Strong Connectivity (SC): $\vec{G}_{r}$ must be strongly connected.

- Broadcast (B): given a source node $s, \vec{G}_{r}$ must contain an arborescence rooted at $s$.

Observe that the properties are listed here in order of decreasing strength, i.e. each feasible range assignment for one problem is also a feasible range assignment for the next problem.

From now on, by Connectivity, Strong Connectivity or BroAdcast, we mean the respective optimization problem, i.e. the problem to find a range assignment $r$ with the respective property that has minimal $\operatorname{cost}(r)$ among all range assignments satisfying this property. E.g., Strong Connectivity is a relaxation of Connectivity.

One can also consider each of these problems with an additional integer parameter $h$, which indicates the maximal number of hops a message may make on its way from one station to another, i.e. there must be a path of length at most $h$ from $s$ (resp. all nodes) to each other. We do not address this problem here, which can also be regarded as setting $h=n-1$.

In this context, the notion of well-spread instances in 2-d was defined in [7], and approximation results for constant $h$ for these special instances were shown therein. Define

$$
\begin{aligned}
D(S) & =\max \{\operatorname{dist}(u, v) \mid u, v \in S\} \\
\delta_{s}(S) & =\min \{\operatorname{dist}(s, v) \mid v \in S \backslash\{s\}\} \\
\delta(S) & =\min \left\{\delta_{s}(S) \mid s \in S\right\}
\end{aligned}
$$

As in [7], we say that a family $\mathcal{S}$ of 2-dimensional instances is well-spread if there exists some positive constant $c$ such that, for any $S \in \mathcal{S}, \delta(S) \geq c D(S) / \sqrt{|S|}$ holds. A possible generalization for other dimensions is to call a family $\mathcal{S}$ of $d$-dimensional instances wellspread if there exists some positive constant $c$ such that, for any $S \in \mathcal{S}, \delta(S) \geq c D(S) / \sqrt[d]{|S|}$ 
holds. Orthogonal regular grids of full dimension are the prototypical well-spread instances. In the following, we sometimes omit the specific set of stations $S$ if it is clear from the context which $S$ is meant.

We are going to use reductions from Vertex Cover problems in graphs with low degree. A vertex cover of a graph $G=(V, E)$ is a subset $V^{\prime} \subseteq V$ of its nodes that contains at least one endpoint of each edge $e \in E$. The VERTEx COVER problem (VC for short) is to find a vertex cover of minimum cardinality. VC is among Karp's original NP-complete problems [11]. It remains hard on various restricted graph classes: Let $k$-VERTEx Cover (or $k$-VC for short) denote the Vertex Cover problem on graphs with maximum degree $k$. $k$-VC is APX-hard for $k \geq 3$ [14]. Planar VC remains NP-complete (in fact even Planar $k$-VC for $k \geq 3$ ) [9], but admits a PTAS [1]. In [2], Berman and Karpinski gave the first explicit inapproximability results for low degree VC. Quite some progress has been made since then, and we are going to use the inapproximability results for 3- $\mathrm{VC}, 4-\mathrm{VC}$ and 5 - $\mathrm{VC}$ by Chlebík and Chlebíková [4], which are (to our knowledge) currently the best such results for these problems.

\subsection{Previous work and our contribution}

For an introduction to this subject, we refer the reader to the survey [6], which to our knowledge is still the current state of affairs on this subject, at least the hardness results presented there. In table 1, previously known and our results for these problems for certain ranges of $\alpha$ and $d$ are listed. New results in this paper are listed in bold print. Note that in the literature, the distance-power gradient $\alpha$ is often implicitly assumed to be an integer. In this paper, we allow $\alpha$ to be any positive real number.

All APX-hardness results, both in [7] and this article, are obtained by reductions from low degree Vertex Cover problems. An entry $\rho$ means that no $\rho$-approximation algorithm can exist for the respective optimization problem, unless $\mathrm{P}=\mathrm{NP}$. The actual numbers given here are obtained by combining our resp. the construction in [7] with the inapproximability results for 3-, 4- resp. 5-VC by Chlebík and Chlebíková [4].

The first NP-hardness result for 3-d Range Assignment Problems was given by Kirousis et al in [12]. Clementi et al [7] showed NP-hardness in 2-d and APX-hardness in 3-d of $\mathrm{SC}$ for $\alpha \geq 2$. It was noticed by Calinescu et al [3] that their constructions also work for C. In [5], these construcions were modified to prove NP-hardness of B.

In this paper, we prove NP-hardness of $\mathrm{C}$ and $\mathrm{SC}$ for all constants $\alpha>0$, and APXhardness of $\mathrm{C}, \mathrm{SC}$ and $\mathrm{B}$ for all $\alpha>1$. Note that B is trivially in $\mathrm{P}$ for $0<\alpha \leq 1$, as setting $r_{s}=\max _{v \in S}\{\operatorname{dist}(s, v)\}$ and $r_{v}=0$ for $v \neq s$ is an optimal range assignment for this problem.

The case $\alpha=1$ for SC, i.e. SC with the Euclidean distance, was formulated as an open problem in [6]. ${ }^{1}$ Another open question in the same survey was if there exists a PTAS for $\mathrm{B}$ in general dimension, which we answer negatively here by giving the first APX-hardness

\footnotetext{
${ }^{1}$ In the PhD-thesis of G. Rossi [15], it is stated without proof that the results in [7] can be applied also when $\alpha=1$.
} 


\begin{tabular}{|c|c|c|c|c|}
\hline & $d=2(\mathrm{old})$ & $d \geq 3($ old $)$ & $d=2($ new $)$ & $d \geq 3($ new $)$ \\
\hline $\mathrm{C}, 0<\alpha \leq 1$ & - & - & $\begin{array}{l}\text { NP-hard } \\
\text { (also for w.s.i.) }\end{array}$ & $\begin{array}{l}\text { NP-hard } \\
\text { (also for w.s.i.) }\end{array}$ \\
\hline C, $\alpha>1$ & \multirow[t]{2}{*}{ - $^{*}$} & \multirow[t]{2}{*}{ - $^{*}$} & \multirow[t]{2}{*}{$\begin{array}{l}\text { NP-hard } \\
\text { (also for w.s.i.) }\end{array}$} & $\begin{array}{l}\text { APX-hard } \\
\left(\rho=1+\frac{1}{260}\right)\end{array}$ \\
\hline C, $\alpha>d$ & & & & (also for w.s.i.) \\
\hline SC, $0<\alpha<1$ & - & - & \multirow{3}{*}{$\begin{array}{l}\text { NP-hard } \\
\text { (also for w.s.i.) }\end{array}$} & $\begin{array}{l}\text { NP-hard } \\
\text { (also for w.s.i.) }\end{array}$ \\
\hline $\mathrm{SC}, \alpha=1$ & - & \multirow[b]{2}{*}{ NP-hard [12] } & & $\begin{array}{l}\text { NP-hard } \\
\text { (also for w.s.i.) }\end{array}$ \\
\hline SC, $1<\alpha<2$ & - & & & $\begin{array}{l}\text { APX-hard } \\
\left(\rho=1+\frac{\sqrt{2}^{\alpha}-1}{\left(7-\sqrt{2}^{\alpha}\right) \cdot 52}\right)\end{array}$ \\
\hline SC, $\alpha \geq 2$ & \multirow[t]{2}{*}{ NP-hard [7] } & \multirow[t]{2}{*}{$\begin{array}{l}\text { APX-hard [7] } \\
\left(\rho=1+\frac{1}{495}\right)\end{array}$} & \multirow[t]{2}{*}{$\begin{array}{l}\text { NP-hard } \\
\text { (also for w.s.i.) }\end{array}$} & $\begin{array}{l}\text { APX-hard } \\
\left(\rho=1+\frac{1}{260}\right)\end{array}$ \\
\hline $\mathrm{SC}, \alpha>d$ & & & & (also for w.s.i.) \\
\hline B, $1<\alpha<2$ & - & - & $\begin{array}{l}\text { NP-hard } \\
\text { (also for w.s.i.) }\end{array}$ & \multirow[t]{2}{*}{$\begin{array}{l}\text { APX-hard } \\
\left(\rho=1+\frac{1}{50}\right)\end{array}$} \\
\hline B, $\alpha \geq 2$ & \multirow[t]{2}{*}{ NP-hard [5] } & \multirow[t]{2}{*}{ NP-hard [5] } & \multirow[t]{2}{*}{$\begin{array}{l}\text { NP-hard } \\
\text { (also for w.s.i.) }\end{array}$} & \\
\hline $\mathrm{B}, \alpha>d$ & & & & (also for w.s.i.) \\
\hline
\end{tabular}

( ${ }^{*}$ NP-hardness for $\alpha \geq 2$ is implicit in [7].)

Table 1: List of previous and new results for different Range Assignment Problems

result for this problem in 3-d. When $\alpha \gtrsim 1$, the term for $\rho$ in the APX-hardness result for $\mathrm{SC}$ remains larger than $1+\frac{1}{702}$. We can also for the first time provide NP-hardness results for well-spread instances for these problems, and, for $\alpha>d$, the first APX-hardness results for these problems.

An important aspect is that our constructions are kept quite simple, especially when compared to the constructions that were used to show the earlier results. In fact, we are mostly reusing the techniques Garey and Johnson employed to give the first NP-hardness proof of the Rectilinear Steiner tree problem in the plane [9]. The notion of a "simple reduction" is of course far from being well defined, but let us try to give some evidence that our reductions are simple indeed: As in [7] and [12], we use reductions from low degree VC problems, and, at some point, we have to construct an orthogonal drawing of a graph in 2-d resp. 3-d. Then, the edges of the drawing have to be replaced by a certain arrangement of points. [7] and [12] use quite technically involved gadgets which have to be placed carefully in the available space. We always replace a line by equally spaced points located on this line, with possibly some distance left free at one or both ends of the line.

Clementi et al [7] use a reduction from 3-VC to SC in 3-d, and it is not clear how this construction should work for graphs with higher degree. Our improved non-approximability factors benefit from the simplicity of our construction: Instead of reducing from 3 -VC, we 
can also use reductions from 4- and 5-VC, for which better inapproximability results have been proven in [4].

The paper is organized as follows: We first review the techniques of Garey and Johnson, and adapt their reduction for our purposes. In the sections thereafter, we describe the particular reduction for the different Range-Assignment problems that we address here.

\section{The reduction of Garey and Johnson}

Given a finite set of points $S$ lying the real plane $\mathbb{R}^{2}$, the Rectilinear Steiner Tree problem seeks to find a tree interconnecting $S$ using only horizontal and vertical lines of shortest possible total length. In 1977, Garey and Johnson published the first proof of the NPhardness of this problem [9]. They also needed to employ the result of Garey, Johnson and Stockmeyer that the planar version of Vertex Cover still remains NP-hard, which was published one year earlier [10].

The line of reductions in [9] is the following:

Planar VC $\rightarrow$ Planar 3-VC $\rightarrow$ Planar Connected 4-VC $\rightarrow$ Rectilinear Steiner Tree Problem in $\mathbb{R}^{2}$

where a connected vertex cover is a vertex cover whose node set induces a connected graph.

In the last reduction of this line, an orthogonal drawing of a planar graph with maximum degree 4 is needed; quite efficient methods for this have been developed in graph drawing. Roughly speaking, in an orthogonal drawing of a graph $G$, all vertices have integer coordinates, all edges are represented by polylines (i.e. piecewise axis-parallel lines between points with integer coordinates), and polylines intersect only at their end-vertices.

Lemma 1. It is possible to efficiently construct polynomial orthogonal drawings of planar graphs with maximum degree 4 in 2-d and arbitrary graphs with maximum degree 6 in 3-d with maximum edge-length $O(n)$.

In fact, in [8] a 3-d orthogonal drawing with maximum edge length $O(\sqrt{n})$ is constructed, and further references in this field can be found therein.

Let us now take a closer look at the reduction from Planar 3-Vertex Cover to Planar Connected 4-Vertex Cover. Variations of this step will be included in all later proofs.

Maybe this construction is best explained by a picture. Figures 1 and 2, taken from [9], show a planar drawing $D$ of an example instance of $3-\mathrm{VC}$ and our reduced Connected 4 -VC instance $\bar{D}$, which is almost but not entirely the same as $D_{G J}$, which we call the graph constructed in [9]. $\bar{D}$ is constructed in the following way (for a rigorous proof, the reader is of course invited to refer to the original proof in [9]):

Let $D=(V, E)$ be a planar graph with maximum degree 3 with a fixed planar embedding. All through this paper, $n=|V|$ and $m=|E|$ are the number of vertices resp. edges of the original VC-instance $D$. 


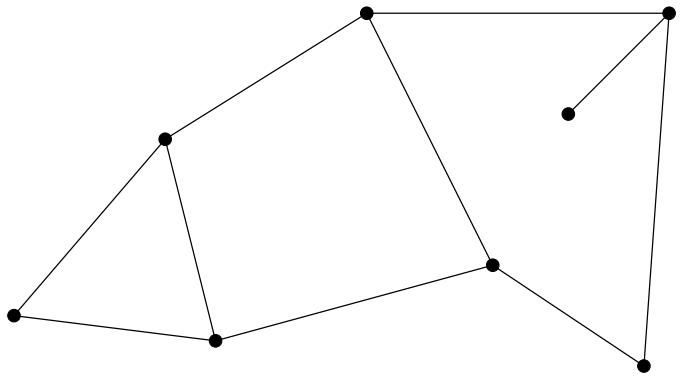

Figure 1: An instance $D$ of $3-\mathrm{VC}$

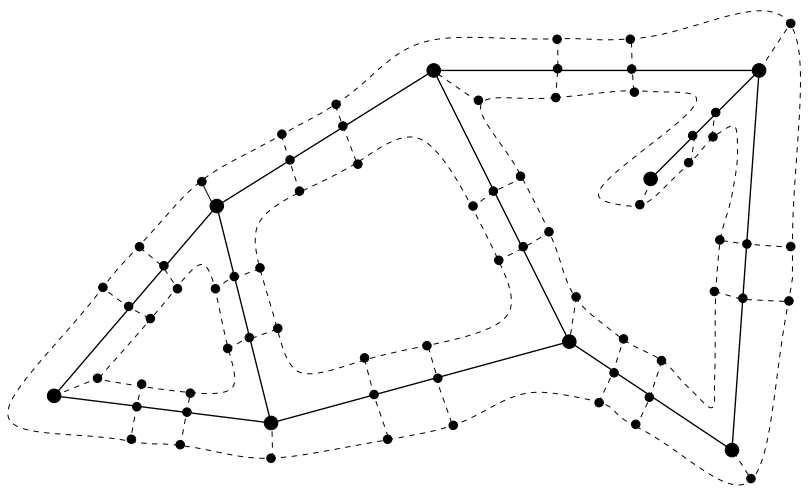

Figure 2: The reduced Connected 4-VC instance $\bar{D}$. Backbone edges are dashed.

- First, split each edge $e=\{x, y\} \in E$ into three edges $\left\{x, x_{e}\right\},\left\{x_{e}, y_{e}\right\}$ and $\left\{y_{e}, y\right\}$ by adding two new vertices $x_{e}, y_{e}$ per edge. Call those new vertices $C$ ("connectors") and the split edges $E^{\prime}$. We call this intermediate graph $D^{\prime}=\left(V \cup C, E^{\prime}\right)$.

- For each vertex $c \in C$, place one new vertex $b_{c, R}$ in each adjacent region $R$ (one or two), and connect $b_{c, R}$ to $c$. For each vertex $v \in V$ of the original graph, place one new vertex $b_{v, R}$ in any neighboring region $R$, and connect $b_{v, R}$ to $v$.

- In each region $R$, connect all vertices $b_{\cdot, R}$ by a walk along the border of the region, like in figure 2. Collect the additional edges of this and the previous step in the set $\bar{E}$, and the nodes in the set $B$ ("backbone").

Now we have described the construction of the planar graph $\bar{D}=\left(V \cup C \cup B, E^{\prime} \cup \bar{E}\right)$ with a fixed embedding.

The following lemma is implicit in the construction of Garey and Johnson:

Lemma 2. D has a vertex cover of size $k \Leftrightarrow D^{\prime}$ has a vertex cover of size $k+m$.

Proof. Let $N$ be a vertex cover of $D$. Let $M=\left\{x_{e} \mid e=\{x, y\} \in E, y \in N\right\} \cup\left\{y_{e} \mid e=\right.$ $\{x, y\} \in E, y \notin N\}$. Now $|M|=m$, and $N \dot{\cup} M$ is a vertex cover of $D^{\prime}$.

Conversely, let $N^{\prime}$ be a vertex cover of $D^{\prime}$, and let $M^{\prime}=\left\{x \mid e=\{x, y\} \in E, x_{e}, y_{e} \in N^{\prime}\right\}$. Now $N=N^{\prime} \cap V \cup M^{\prime}$ is a vertex cover of $D$, and has at least $m$ nodes less than $N^{\prime}$.

The constructed graph $\bar{D}$ that we present here is in fact slightly different from the graph $D_{G J}$ constructed in [9]: In $D_{G J}$, each backbone node $b \in B$ has a "spike", i.e., it is additionally connected to a copy of itself which has no other neighbor. The single purpose of these spikes is to ensure that, wlog., the whole set of backbone nodes $B$ is included in every vertex cover of $\bar{D}$. Additionally, one connector per edge in $D$ must lie in every vertex cover, providing connectivity of the backbone. As every node has a backbone neighbor, all feasible vertex covers for $\bar{D}$ are wlog. connected. So the size of a minimum vertex cover for $D$ is $k$ iff a minimum connected vertex cover of $D_{G J}$ has size $k+m+|B|$. 
In the context of range assignments for radio stations, we will not need these spikes to ensure that the backbone is connected.

\section{Hardness results for CONNECTIVITY}

\subsection{NP-hardness of CONNECTIVITY in 2-d}

After this preparatory work, which is exactly the same as in [9] (except for leaving out the spikes), we complete the construction for our first Range Assignment Problem:

- As in [9], construct an orthogonal drawing of $\bar{D}$ in the plane.

- Scale the whole drawing by factor 3 .

- Replace each line in the drawing by a set of equidistant points in the following way: Place one station at one end of the line, and:

- For each polyline representing an edge originally in $E^{\prime}$, place stations on every point with integer coordinates, i.e. points at distance one.

- For each line representing some part of a backbone edge (those in $\bar{E}$ ), place stations at distance $\frac{3}{4}$.

By scaling by a factor $c$ we mean multiplying the coordinates of each point with $c$. By a vertex (or node) station we mean a station representing a node of the graph $D^{\prime}$. By an edge-end we mean the last station on an edge before the vertex station.

Note that because of the scaling step, the first and last station on a straight line segment always have integer coordinates, and the minimum distance between two vertex stations is 3. See figures 3 and 4 for an illustration of this reduction.

Originally given a planar instance $D$ of 3 -Vertex Cover with a fixed embedding, we have constructed a blown-up version $\bar{D}$ and, by our last step, associated a set of points $S$ in the plane with $D$. We now claim that a solution for the CONNECTIVITY problem for $S$ also automatically yields a minimal vertex cover for $D$.

Theorem 3. For any $\alpha>0$, Connectivity in $\mathbb{R}^{2}$ is NP-hard.

Before we begin with the proof of this theorem, we formulate a simple lemma, which is fundamental in the hardness proofs for the Connectivity and Strong Connectivity types of problems.

Lemma 4. In a range assignment $r$ for a set of stations $S$ that satisfies the CONNECTIVITY or the Strong ConneCtivity property, $r_{s} \geq \delta_{s}(S)$ must hold for all $s \in S$.

Proof. If there was a station $s$ with $r_{s}<\delta_{s}(S)$, it could not send data to any other station, meaning that there cannot be an arc/edge leaving $s$. So $G_{r}$ cannot be connected, and $\vec{G}_{r}$ cannot be strongly connected, a contradiction. 


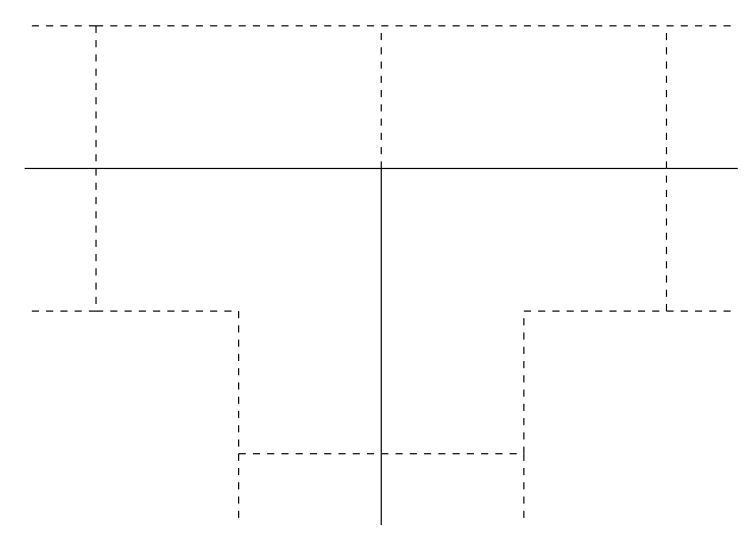

Figure 3: A small part of an orthogonal drawing of a graph $\bar{D}$.

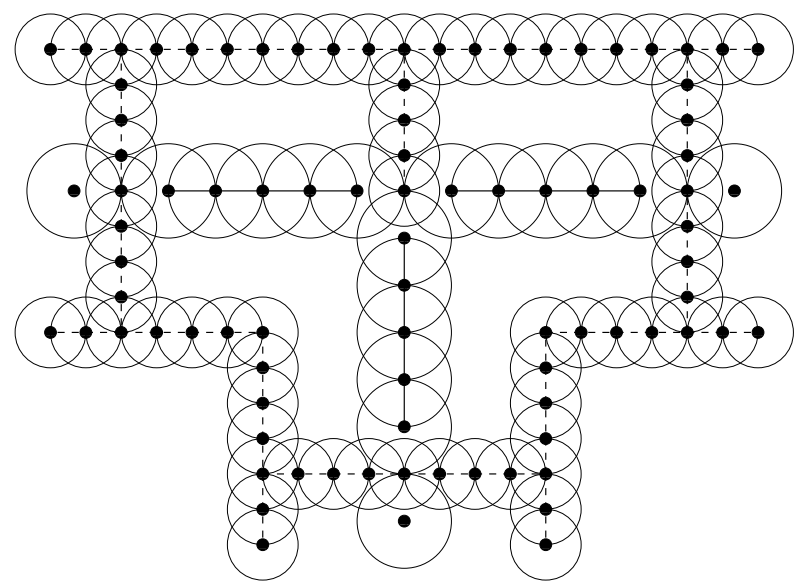

Figure 4: The resulting set of stations, with a minimal configuration and the induced connectivity graph.

As a direct consequence of Lemma 4, it makes sense to define a minimal configuration $r_{\text {min }}$ in the following way: For all $s \in S$, set $r_{\min }(s)=\delta_{s}$. A feasible range assignment $r$ for Connectivity or Strong Connectivity will satisfy $r \geq r_{m i n}$.

Proof. of Thm. 3: Let us look at the minimal configuration $r_{\text {min }}$ for $S$ : All stations on edges in $E^{\prime}$ have $r_{\text {min }}=1$, and all stations on edges in $\bar{E}$, the backbone edges, have $r_{\min }=\frac{3}{4}$. As all intersections (i.e. stations representing the nodes of $\bar{D}$ ) have at least one adjacent edge from the backbone, all those stations also have $r_{\min }=\frac{3}{4}$.

Observe that the undirected communication graph of the minimal configuration, $G_{r_{\text {min }}}$, already has quite large connected components (cf. fig. 4): There is one connected component corresponding to each edge in $E^{\prime}$, and the backbone is one connected component in the minimal configuration. For notational convenience, we refer to a component corresponding to an edge $e \in E^{\prime}$ as an edge-component, or the e-component. We also use the terms incident or adjacent with edge-components or vertex stations when we mean that the corresponding edges or vertices have this property.

Let $M=\operatorname{cost}\left(r_{\text {min }}\right)$ be the cost of the minimal configuration, and let $k$ be the number of vertices in a minimal vertex cover for $D$. We claim that a minimal range assignment with property ConneCTIVITY has cost $M+\gamma(m+k)$, where $\gamma=1-\left(\frac{3}{4}\right)^{\alpha}$, which implies the NP-hardness of CONNECTIVITY.

To prove our claim, we argue that, without loss of generality, in a minimal range assignment, only radii of node stations are increased, and those that are increased are increased by $\frac{1}{4}$, resulting in extra energy consumption $\gamma$.

First, we want to rule out that non-adjacent edge-components are directly connected to the same station. Assume conversely that $l$ non-adjacent edge-components were connected via the same station. This means there would be $l$ stations ${ }^{2} S^{\prime}$ with radii increased from

\footnotetext{
${ }^{2}$ possibly even $l+1$, if they are connected via a backbone station
} 
$r_{\text {min }} \leq 1$ to at least to some value $C>1$, costing at least $l\left(C^{\alpha}-1\right)$. Instead, we could have connected those $l$ edges to the backbone by increasing at most $l$ radii to from $\frac{3}{4}$ to 1 . In order for the first increase being cheaper,

$$
l\left(C^{\alpha}-1\right) \leq l\left(1-\left(\frac{3}{4}\right)^{\alpha}\right) \Longleftrightarrow C^{\alpha}+\left(\frac{3}{4}\right)^{\alpha} \leq 2
$$

would have to hold. As $x+1 / x \geq 2 \forall x>0$, this would imply that $C \leq \frac{4}{3}$, which means that some radii are increased to not more than this quantity. But by construction, it is not possible to connect non-adjacent edges with such a low radius, a contradiction to the assumption that all $l$ edges are directly connected.

When an edge-component (or several adjacent edge-components) is connected to some node in the backbone, the cheapest way to achieve this is obviously to increase the radius of the station representing a node incident to it from $\frac{3}{4}$ to 1 (see also fig. 6). This is always cheaper than connecting adjacent edge-components directly:

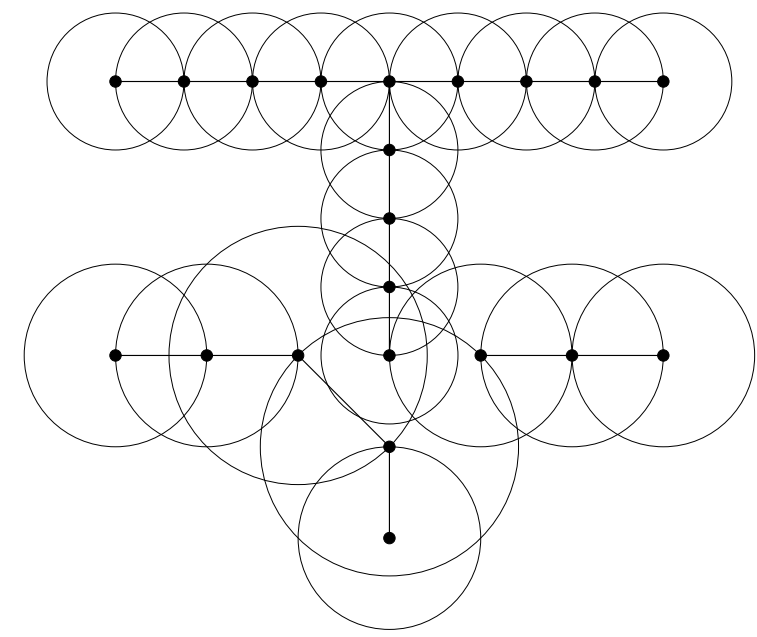

Figure 5: An unwanted connection scenario.

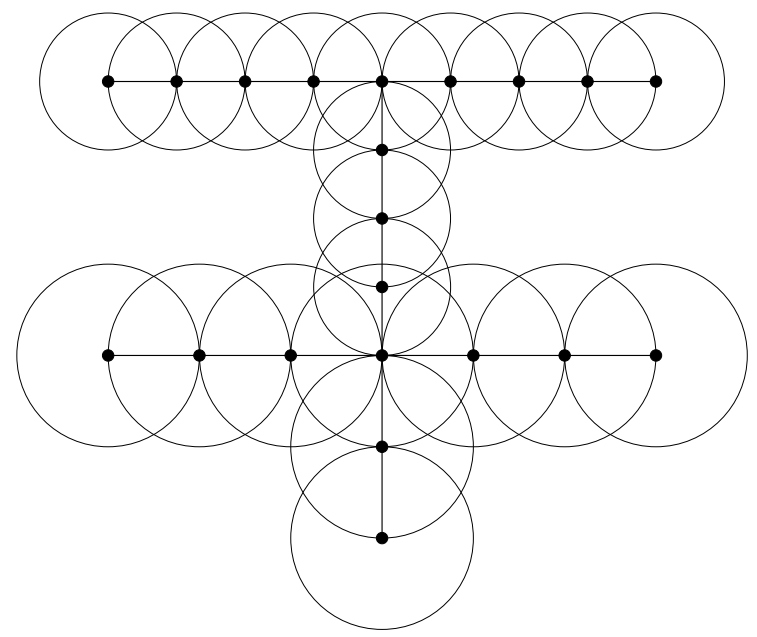

Figure 6: The cheapest way to connect edges. Automatically, all incident edges are attached.

Assume that two nodes on non-backbone edges would have increased radii in order be directly connected. The cheapest case of this would be if two adjacent such edges would have their last node's radius increased from 1 to $\sqrt{2}$ (see figure 5 ). To rule out this case (and in consequence, all other more expensive cases), we have to show that

$$
\begin{aligned}
\delta=1-\left(\frac{3}{4}\right)^{\alpha} & \leq 2\left(\sqrt{2}^{\alpha}-1\right) \\
\Leftrightarrow 3 & \leq 2 \sqrt{2}^{\alpha}+\left(\frac{3}{4}\right)^{\alpha}
\end{aligned}
$$

which is easy:

$$
2 \sqrt{2}^{\alpha}+\left(\frac{3}{4}\right)^{\alpha}>2 \sqrt{2}^{\alpha}+\frac{1}{\sqrt{2}^{\alpha}}>\sqrt{2}^{\alpha}+2>3 .
$$


We have now argued that in a minimal solution to CONNECTIVITY, only radii of stations corresponding to nodes of $D^{\prime}$ are increased from the minimal configuration, and if so, they are increased from $\frac{3}{4}$ to 1 . Note that for nodes where this is the case, all incident edges are thereby connected to the backbone and its connected component (cf. fig. 6). So in order for the range assignment to be connected, these nodes have to form a vertex cover of $D^{\prime}$. On the other hand, by virtue of the backbone, increasing each node in a vertex cover for $D^{\prime}$ already makes a minimal configuration connected.

Finally, we notice that by Lemma 2, a minimal vertex cover for $D^{\prime}$ is of size $m+k$, which means that a minimal range assignment with ConNECTIVITY has cost $M+\gamma(m+k)$, which concludes this proof.

\subsection{NP-hardness for well-spread instances}

Notice that the construction above is already "nearly" well-spread: The minimal distance is a constant. So each set of stations on a straight line segment already is a well-spread instance in $\mathbb{R}$. But for $S$ being well-spread (in $\mathbb{R}^{2}$ ), there may be "too few" points in $S$. In order to ensure that the construction is well-spread we fill it up in such a way that the additional stations play no role in the reduction, i.e. they are never increased above their minimal distance in a minimal solution, and so only contribute to the cost of the minimal configuration.

To do so, we use a really cheap trick: By construction, the outer face is bounded completely by backbone edges, all lying on a grid with mesh distance $\frac{3}{4}$. We now simply "fill up" the outer face with stations of this grid in such a way that the outer stations of this grid form a square of some desired size. For instance, twice the maximum of the width and height of the drawing would suffice to produce well-spread instances with constant $c \geq \frac{3}{4} \frac{1}{\sqrt{2}}$. Obviously, in a minimal configuration the additional vertices all have radius $\frac{3}{4}$ and all are in the same connected component as the backbone, and it does not make sense to increase any of their radii. It is obvious how to fill up such a well-spread square to a well-spread cube.

Theorem 5. For any $\alpha>0$, ConneCtivity on well-spread instances in $\mathbb{R}^{2}$ and $\mathbb{R}^{3}$ is NP-hard.

\subsection{APX-hardness of ConNeCTIVITY in 3-d}

The construction in the NP-hardness proof for CONNECTIVITY is far from being approximation-preserving: The fixed cost $M$ of the minimal configuration is far larger than the variable cost of the vertex cover. More precisely, $M$ would have to be bounded by some (preferably low) constant factor times the number of vertices $n$. To the best of our knowledge, no orthogonal 3-d graph drawing method is known that uses only $O(n)$ total length, so we cannot hope to achieve this goal with this construction when $\alpha \leq 1$. However, the 
situation changes when $\alpha>1$ : Because the power function is now strictly convex, smaller radii cost far less than big radii. This means we can make the power of the internal radii on the edges negligible by inserting a large number of stations on every edge at a very small distance. A polynomial number of stations suffices. This fact has already been exploited by Clementi et al in their constructions [7].

Lemma 6. Let $L$ be the total length of all $m$ edges in a polynomially scaled orthogonal drawing of $\bar{D}$. Suppose we replace one length unit of a line by $s+1$ stations at distance $1 / s$. Then the total power consumption of a minimal configuration $r_{\min }$ amounts to

$$
M=(L s+n-m) \cdot \frac{1}{s^{\alpha}} .
$$

So if $\alpha>1$, we can make $M<c$ for any constant $c>0$ with a polynomial number of stations.

Proof. The term for $M$ is obvious, where the term $n-m$ avoids double counting. Note that, because wlog. $\bar{D}$ is connected, and vertex cover on a tree is trivial, we can assume this term is non-positive. So we have $M \leq L s^{1-\alpha}$, and if $\alpha>1$, we can make this term smaller than any constant with a number $s$ polynomial in $L$, which again is polynomial in the input. This will result in a total of $L s$ stations, which remains polynomial.

Before we begin with the construction, we state the results of Chlebík and Chlebíková [4] for the non-approximability of 3-, 4- and 5-Vertex-Cover, which are to our knowledge currently the best results for these problems.

Lemma 7 (Chlebík and Chlebíková, [4]). It is NP-hard to approximate the solutions of:

- 3-Vertex Cover to within $1+\frac{1}{99}$,

- 4-Vertex Cover to within $1+\frac{1}{52}$,

- 5-Vertex Cover to within $1+\frac{1}{50}$,

even on 3-, 4-resp. 5-regular graphs.

Given a low-degree instance of Vertex Cover $D=(V, E)$, we describe now how to build the graph $\bar{D}$ which later gets drawn in the Euclidean space $\mathbb{R}^{3}$. Note that we cannot use our reduction to prove APX-hardness of Range Assignment Problems in 2-d, because Planar Vertex Cover is not APX-hard, but there exists a PTAS for this problem [1]. This time, as we do not have to observe planarity, the construction of the backbone becomes very simple: Let the vertices of $V$ be given in some arbitrary order $V=\left\{v_{1}, \ldots, v_{n}\right\}$. The backbone vertices $B$ contain one copy of each original vertex: $B=\left\{v_{1}^{\prime}, \ldots, v_{n}^{\prime}\right\}$, and the backbone edges consist of one edge between each original node and its copy, and a cycle through all backbone nodes: $\bar{E}=\left\{\left\{v_{i}, v_{i}^{\prime}\right\} \mid 1 \leq i \leq n\right\} \cup\left\{\left\{v_{i}^{\prime}, v_{i+1}^{\prime}\right\} \mid 1 \leq i \leq n-1\right\} \cup\left\{\left\{v_{n}^{\prime}, v_{1}^{\prime}\right\}\right\}$. Call $\bar{D}=(V \cup B, E \cup \bar{E})$. Given a constant $0<\varepsilon<1$, choose $s$ according to Lemma 6 and construct a polynomial set of stations $S$ in the following way: 
- Construct an orthogonal drawing of $\bar{D}$ in $\mathbb{R}^{3}$.

- Scale the drawing by factor 3 .

- For all polylines representing original edges $e \in E$, remove the first and last open unit interval of the polyline (i.e. do not erase any integer points).

- Replace all remaining unit line segments with $s+1$ stations along this line at distance $1 / s$.

Here, the scaling step is needed to ensure that at least one length unit of each edge remains. Note that when the maximum degree in $D$ is $\Delta$, the maximum degree of $\bar{D}$ will be $\Delta+1$. So according to Lemma 1 , we must have $\Delta \leq 5$. We use this set of stations in order to prove

Theorem 8. For any $\alpha>1$, it is NP-hard to approximate CONNECTIVITY in $\mathbb{R}^{3}$ within $1+\frac{1}{260}$.

Proof. Again, we first consider a minimal configuration $r_{\text {min }}$ of $S$ with cost $M$ and its communication graph $G_{r_{\text {min }}}$. Due to the construction, all stations on backbone edges already lie in one connected component, and there is one component for each original edge. Similar arguments as in the proof for Theorem 3 show that in a minimal solution, all edges are directly connected to the backbone, and only vertex stations may have an increased radius of 1 . In order to be connected, the nodes corresponding to these vertex stations must form a vertex cover for $D$. Additionally, in each edge component, one of the two end stations' radii must be increased to 1 . On the other hand, we can always take one station which is close to a station from the node cover and whose radius thus is increased to 1 , leading to a connected range assignment.

Note that because $\varepsilon<1$, we have shown that there exists a node cover of size at most $k$ for $D$ iff there is a range assignment $r$ for $S$ costing no more than $k+m+\varepsilon$. In a graph with maximum degree $\Delta$, the size of a vertex cover is at least $m / \Delta$. We now assume that $D$ has maximum degree 4 , and due to the choice of $s$ we have that

$$
\operatorname{cost}(r) \leq M+k+m \leq 5 k+\varepsilon
$$

So if we could approximate Connectivity in 3 -d to $1+\frac{1}{5 \cdot 52}$, this would allow us to construct a vertex cover of size at most

$$
\left\lfloor k+\frac{1}{5 \cdot 52}(5 k+\varepsilon)\right\rfloor=\left\lfloor\left(1+\frac{1}{52}\right) k+\frac{\varepsilon}{260}\right\rfloor \leq\left(1+\frac{1}{52}\right) k,
$$

i.e. we could approximate 4 -Vertex Cover up to $1+\frac{1}{52}$. According to Lemma 7 , this would solve an NP-hard problem.

Note that we have chosen to reduce from 4-Vertex Cover, because this optimizes the trade-off between inapproximability result and reduction size. 


\subsection{APX-hardness for well-spread instances}

This idea behind this proof is basically the same as in the NP-hardness proof for wellspread instances: We fill up the surrounding space with a grid of mesh distance $\delta$. Note that in order to stay approximation preserving, the additional stations must not be too expensive.

Theorem 9. For any $\alpha>d \geq 3$, approximating ConNECTIVITY within $1+\frac{1}{260}$ remains NP-hard even when restricted to well-spread instances.

Proof. We deal here with the most interesting case, $d=3$, but everything holds also for higher dimensions.

Let $a$ be the largest of width, height and depth of the orthogonal drawing of the reduced Vertex Cover instance $\bar{D}$. In order to proof the claim, we show that it is possible to construct a cubical grid of stations $C$ with side length $2 a$ having a connected range assignment costing no more than any constant $\varepsilon$ in polynomial time.

We will place stations on this grid at distance $1 / s$, so this cube will have $(2 a s+1)^{3}$ stations, and its minimal range assignment $r_{\min }$, which is already connected, will have cost

$$
\operatorname{cost}\left(r_{\min }\right)=\frac{(2 a s+1)^{3}}{s^{\alpha}}
$$

Clearly, for constants $\alpha>3$ and $\varepsilon<1$ we can choose a polynomial $s$ in such a way that $\operatorname{cost}\left(r_{\min }\right)<\varepsilon$. Note that this cubical grid is well-spread, even when we cut out a cube of sidelength $(a+1)$, and insert the set of stations constructed for $\bar{D}$ in the APXhardness proof, with $s$ as in the cube. Finally, we connect an arbitrary edge near to the grid stations with a line of stations at distance $1 / s$ with the cut-out cube. Surely, the minimal configuration for this new set of stations will cost less than the one of whole cube, so the equations from the above proof will still hold, implying the claimed result.

This construction for APX-hardness of well-spread instances will work for all problems.

\section{Hardness results for Strong Connectivity}

In the remainder of this article, we will adapt our reductions for ConNECTIVITY for the Strong Connectivity and Broadcast problems.

\subsection{NP-hardness}

In fact, here the reduction will be exactly the same as for CONNECTIVITY, but the proof will slightly differ. The main difference is that now directed links are established already when one of the two stations has a large enough radius.

Theorem 10. For any $\alpha>0$, Strong Connectivity in $\mathbb{R}^{2}$ is NP-hard, already for well-spread instances. 


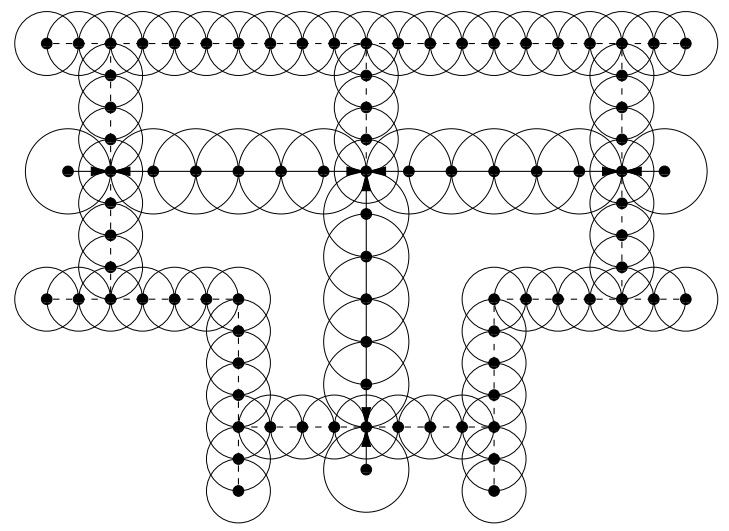

Figure 7: The directed communication graph of $r_{\text {min }}$.

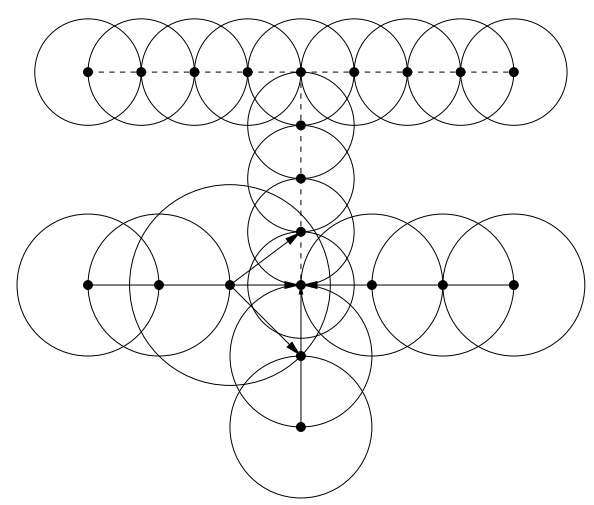

Figure 8: Now already one radius of $\sqrt{2}$ would suffice.

Proof. The minimal configuration $r_{\text {min }}$ due to Lemma 4 is of course the same as in the proof of Theorem 3, but its directed communication graph $\vec{G}_{r_{m i n}}$ is a bit different: The strongly connected components are the same as the connected components in $G_{r_{m i n}}$ (namely one for the whole backbone and one for each split edge), but additionally, each split edge component already has one two outgoing arcs, one to each incident vertex station. (cf. fig. 7) Still, only vertex stations can have increased radii in a minimal solution: The cheapest alternative now increases one edge-end from 1 to $\sqrt{2}$ (see fig. 8), with additional cost $\sqrt{2}^{\alpha}-1$ instead of $1-\left(\frac{3}{4}\right)^{\alpha}$, which, for all $\alpha$, saves us nothing. And if $\alpha \geq 1$, it is obvious that no radius is increased to more than 1 .

The situation changes here when $\alpha<1$ : As links do not have to be bidirectional, and now the cost function is strictly concave, it could pay off to increase just one radius to some large value $C \gg 1$ to send data to many (or possibly all) stations. To be on the safe side, we scale the instance again by a factor of $\sqrt[\alpha]{n}$, which will ensure that

$$
C^{\alpha}-1 \geq n \gamma
$$

holds for radius $C \geq \frac{4}{3} \sqrt[\alpha]{n}$, meaning that it is already cheaper to increase every original vertex to 1 than one single radius to more than $\frac{4}{3} \sqrt[\alpha]{n}$, which would after scaling not reach any new component.

The proof for well-spread instances is the same as in Theorem 5.

\subsection{APX-hardness}

The only difference to the construction for ConNECTIVITY is that now the outgoing arc of an edge does not have to be parallel to the ingoing arc. If some edge-end is increased to 1 , and the incident vertex is not, it could indeed be cheaper to increase the border station further to $\sqrt{2}$ and send to another adjacent edge. (cf. fig. 9) This could indeed be cheaper if $\alpha<2$, and as this is the only thing we have to worry about, let us assume in the following that $1<\alpha<2$. 
We will now present a slightly changed reduction: To the original Vertex Cover instance $D=(V, E)$, no new vertices but only new edges will be added. For vertex set $V=$ $\left\{v_{1}, \ldots, v_{n}\right\}$, add a directed Hamiltonian cycle $\bar{E}=\left\{\left(v_{i}, v_{i+1}\right) \mid 1 \leq i \leq n-1\right\} \cup\left\{\left(v_{n}, v_{1}\right)\right\}$ as the backbone, already completing the construction of $\bar{D}=(V, E \dot{\cup} \bar{E})$. The direction of the added arcs is needed only for notational convenience later on. Note that $\bar{D}$ may contain parallel backbone and original edges, which is not a problem.

The construction is now similar to the one before; the new thing is we also erase some part of backbone lines:

- Let $\beta=\sqrt[\alpha]{2-\sqrt{2}^{\alpha}}$ and choose an $\varepsilon<1-\beta^{\alpha}$; choose an $s$ according to Lemma 6 .

- Construct a polynomial orthogonal drawing of $\bar{D}$ in $\mathbb{R}^{3}$.

- Scale the drawing by factor 3 .

- For all polylines representing original edges $e \in E$, erase the first and last open unit interval of the polyline (i.e. do not erase any integer points).

- For all polylines representing backbone edges $e \in \bar{E}$, erase the first ${ }^{3}$ open interval of length $\sigma / s$, where $\sigma \in \mathbb{N}$ is chosen such that $(\sigma-1) / s<\beta \leq \sigma / s$. Again, erase neither the first nor the last point of this interval.

- Let $\varepsilon<1-\beta^{\alpha}$, and replace all remaining line segments of length 1 (resp. $1-\sigma / s$ ) with $s+1$ (resp. $s-\sigma+1$ ) stations along this line at distance $1 / s$.

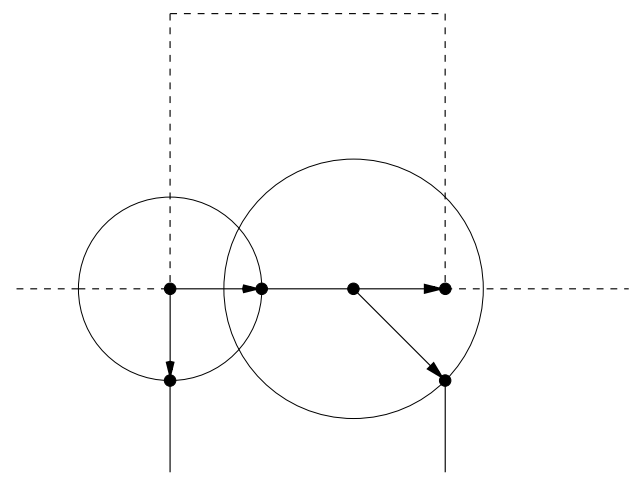

Figure 9: A cheaper way to connect edges when $\alpha<2$.

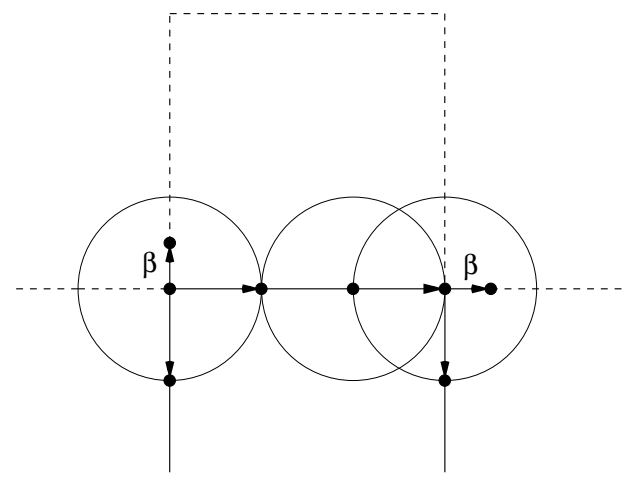

Figure 10: By leaving spaces of length $\beta$ in the backbone, this problem is fixed.

So this time, the minimal configuration consists of one strongly connected component for each original or backbone edge. Note that $0<\beta<2-\sqrt{2}<0.6$. In order for $S$ to be strongly connected, at least one of the end stations of each backbone edge must have radius at least $\beta$; setting all vertex radii to $\beta$ suffices to make the backbone strongly connected. As it has no advantage not to do so, we assume this is the case.

\footnotetext{
${ }^{3}$ i.e. the interval starting at the tail of $e$
} 
This means now every vertex station wlog. already has radius $\beta$. If we would now choose to transmit data with an edge station, we would pay at least additional cost $\sqrt{2}^{\alpha}-1$ instead of paying $1-\beta^{\alpha}$ for an incident vertex station, which due to the choice of $\beta$ is not cheaper (see also fig. 10). So now we can assume that only vertex stations are used for connectivity, and the vertex stations with radius 1 in a minimal range assignment with Strong Connectivity form a minimal vertex cover. This means a minimal range assignment $r$ costs

$$
\operatorname{cost}(r) \leq n \beta^{\alpha}+k\left(1-\beta^{\alpha}\right)+m+\varepsilon
$$

where $k$ is the size of a minimal vertex cover for $D$. As the hardness results in Lemma 7 also hold for regular instances, we can assume that $k \geq m / \Delta=n / 2$. We choose to reduce from 4-Vertex Cover, and normalize the costs for $r$ s.t. the cost for each node in the vertex cover is 1 :

$$
k+\frac{n \beta^{\alpha}+m}{1-\beta^{\alpha}} \leq k+\left(\frac{2+\beta^{\alpha}}{1-\beta^{\alpha}}\right) 2 k+\frac{\varepsilon}{1-\beta^{\alpha}}<\left(\frac{7-\sqrt{2}^{\alpha}}{\sqrt{2}^{\alpha}-1}\right) k+1
$$

which finally concludes the proof of

Theorem 11. It is NP-hard to approximate STROng Connectivity within $1+\frac{1}{260}$, if $\alpha \geq 2$, and within $1+\frac{\sqrt{2}^{\alpha}-1}{\left(7-\sqrt{2}^{\alpha}\right) \cdot 52}$, if $1<\alpha<2$.

\section{Hardness Results for BROADCAST}

The main difference to the two preceding problems is that we cannot make use of Lemma 4 , because no node (except for the source node $s$ ) has to increase its radius a priori. Indeed, the optimal solution when $0<\alpha \leq 1$ is to have $s$ directly broadcast to all stations, and all other stations have radius 0 , so we assume $\alpha>1$. This time, we use the APX-type construction also for proving the NP-hardness results.

Theorem 12. For any $\alpha>1$, BROADCAST in $\mathbb{R}^{2}$ is NP-hard, already for well-spread instances.

Proof. Given an instance of planar 3-Vertex Cover $D$, construct $\bar{D}$ as in Theorem 3, draw this orthogonally in the plane, scale it by factor 3, remove the first and last unit of nonbackbone edges, and replace the lines by an appropriate number of stations s.t. $\varepsilon<1$. Let the source station $s$ be any backbone station. In order to broadcast to all stations, we have to set the vertex stations of a vertex cover of $D^{\prime}$ to radius 1 . This time, we cannot argue with the minimal configuration, but as every (Strong) Connectivity solution is also feasible for BROADCAST, we can be sure that the overhead cost of the construction does not exceed $\varepsilon$. This means there will be a range assignment of size $<m+k+1$ iff there is a vertex cover of $D^{\prime}$ of size $m+k$, showing NP-hardness of BROADCAST.

Again, this construction can be made well-spread with the same method as in Theorem 5 , where the grid now has mesh-distance $1 / s$. Note that despite of the now larger overhead, everything is still polynomial. 
Theorem 13. For any $\alpha>1$, approximating BROADCAST in $\mathbb{R}^{3}$ better than $1+\frac{1}{50}$ is NP-hard.

Proof. We use exactly the same construction as in the proof for Theorem 8, and let the source station be some backbone station. As in the preceding proof, this time exactly the vertex stations of a vertex cover have to be increased to radius 1; no additional edge stations have to be significantly increased. This means a range assignment of size less than $k+\varepsilon$ leads to a vertex cover of size $k$, so we only have overhead $\varepsilon$, which we can make arbitrarily small. So this time it pays to reduce from 5-Vertex Cover, yielding the claimed result.

\section{Open problems and acknowledgements}

It is still an intriguing question if the above problems remain APX-hard in the plane or allow a PTAS. A similar reduction as in this paper from a planar APX-hard problem might produce such a result.

An interesting question in Graph Drawing would be whether graphs of low degree could be drawn with total length in $O(n)$ in 3-d. If so, this would imply APX-hardness of $\mathrm{C}$ and $\mathrm{SC}$ in 3 -d even for $\alpha=1$. On the other hand, a PTAS for this case would imply that such drawings cannot exist, unless $\mathrm{P}=\mathrm{NP}$.

The author would like to thank Walter Kern for suggesting this problem, Dominique Andres and Christoph Buchheim for helpful discussions, Therese Biedl for reference [8] and Marek Karpinski for reference [4].

\section{References}

[1] B. Baker, Approximation Algorithms for NP-Complete Problems on Planar Graphs, Journal of the ACM 41 (1): 153-180, 1994.

[2] P. Berman and M. Karpinski, On Some Tighter Inapproximability Results, in: Proc. 26th ICALP, pp. 200-209, 1999. Also available as ECCC Report TR98-065 at http://eccc.uni-trier.de/eccc/.

[3] G. Călinesc, I. Măndoiu and A. Zelikovsky, Symmetric Connectivity with Minimum Power Consumption in Radio Networks, Proc. IFIP TCS 223, pp. 119-130, 2002.

[4] M. Chlebík and J. Chlebíková, Inapproximability results for bounded variants of optimization problems, Proc. FCT (2003), LNCS 2751: 27-38, 2003. Also available as ECCC Report TR03-026.

[5] A. Clementi, P. Crescenzi, P. Penna, G. Rossi and P. Vocca, A Worst-Case Analysis of an MST-based Heuristic to Construct Energy-Efficient Broadcast Trees in Wireless Networks. Technical Report 010, University of Rome "Tor Vergata", Math. Department, 2001. 
[6] A. Clementi, G. Huiban, P. Penna, G. Rossi and Y. Verhoeven, Some Recent Theoretical Advances and Open Questions on Energy Consumption in Ad-Hoc Wireless Networks, in: Proc. 3rd Workshop on Approximation and Randomization Algorithms in Communication Networks (ARACNE): 23-38, 2002.

[7] A. Clementi, P. Penna and R. Silvestri, On the Power Assignment Problem in Radio Networks, Mobile Networks and Applications 9 (2): 125-140, April 2004. Also available as ECCC Report TR00-054.

[8] P. Eades, C. Stirk and S. Whitesides, The techniques of Kolmogorov and Bardzin for three-dimensional orthogonal graph drawings, Information Processing Letters 60 (2): 97-103, 1996.

[9] M. Garey and D. Johnson, The Rectilinear Steiner Tree Problem is NP-Complete, SIAM Journal of Applied Mathematics 32 (4): 826-834, 1977.

[10] M. Garey, D. Johnson and L. Stockmeyer, Some simplified NP-Complete Problems, Theoretical Computer Science 1: 237-267, 1976.

[11] R. Karp, Reducibility among combinatorial problems, in: R. Miller and J. Thatcher (eds.), Complexity of Computer Computations, 85-103, 1972.

[12] L. Kirousis, E. Kranakis, D. Krizanc and A. Pelc, Power consumption in packet radio networks, Theoretical Computer Science 243: 289-305, 2000.

[13] K. Pahlavan and A. Levesque, Wireless Information Networks, Wiley-Interscience, 1995.

[14] C. H. Papadimitriou and M. Yannakakis, Optimization, approximation, and complexity classes, Journal of Computer and System Sciences 43, 425-440, 1991.

[15] G. Rossi, The Range Assignment Problem in Static Ad-Hoc Wireless Networks (PhDthesis), University of Siena, 2003. 